\title{
The Devil Is in the Details
}

\author{
lan Jenkins, MD and Joseph Vinetz, MD \\ Department of Medicine, University of California, San Diego, San Diego, California.
}

\begin{abstract}
The approach to clinical conundrums by an expert clinician is revealed through presentation of an actual patient's case in an approach typical of morning report. Similar to patient care, sequential pieces of information are provided to the clinician who is unfamiliar with the case. The focus is on the thought processes of both the clinical team caring for the patient and the discussant.
\end{abstract}

A 47-year-old male presented to a community hospital with 5 weeks of daily fevers, accompanied by headache, myalgias, and malaise. He reported that his symptoms began abruptly 2 days after a weekend of camping in Connecticut.

This patient describes the onset of undifferentiated fever 2 days after a weekend of camping. Few infectious diseases have such short incubation periods, and either the accuracy of the history or the relationship of the camping trip to the present illness is thus questionable. However, more information about the onset and nature of the illness, and details about food, animal, water, mud, cave, wood chopping, and other environmental exposures during his trip is required. The exact dates of the camping trip may be helpful, as there is clear seasonality to vector-borne diseases such as Lyme disease, babesiosis, ehrlichiosis, and rickettsial infections. Conditions unrelated to his camping trip, such as malignancies, rheumatologic conditions, and other infectious causes of prolonged fever, such as tuberculosis, endocarditis, or osteomyelitis, are more likely, given the duration of fever.

The fevers were accompanied by chills, without rigors, and subjectively worsened over the first 2 days. At that point, the patient began taking his temperature, and noted fevers of $38.5^{\circ}$ $\mathrm{C}$ to $40^{\circ} \mathrm{C}$ occurring once or twice daily, generally in the afternoon or evening. The patient did not recall tick bites but did not carefully examine himself for ticks; he reported numerous mosquito bites during the trip. The patient camped in a tent and grilled meats and other food he had brought in a cooler. No family members or other travelers became ill. He denied spelunking, but had collected wood for camp fires, and acknowledged swimming in a freshwater pond during his trip, which occurred in August.

West Nile fever, St. Louis encephalitis, and eastern equine encephalitis are transmitted by mosquitoes in New England, but are unlikely causes of prolonged fever. Water exposure suggests the possibility of leptospirosis, and wood exposure suggests blastomycosis, but this usually presents with a pulmonary syndrome. Food-borne illness seems unlikely. While no aspect of the history has pinpointed a specific diagnosis, exploring the progression of symptoms may offer a clue, and if he has undergone any previous evaluation, the results may significantly alter the differential diagnosis. For example, arthritis may develop weeks after fever in adult onset Still's disease, negative blood cultures would lower the probability of endocarditis, and

Address for correspondence and reprint requests: Dr. Ian Jenkins, $200 \mathrm{~W}$ Arbor Drive, San Diego, CA, 92103; Telephone: 619-471-9565; Fax: 619-543-8255; ihjenkins@ucsd.edu. 
common sites of pyrogenic malignancies (eg, liver, kidneys, and especially lymph nodes) may already have been imaged.

During the first 3 weeks of illness, the patient experienced daily fever and a gradual, 10pound weight loss. Over the next 10 days, he sought medical attention at 3 emergency departments. At one, a head computed tomography (CT) showed possible sinusitis, and he was prescribed a 7-day course of clarithromycin, which he took without any improvement. At 2 others, he was told that his laboratory studies, and a CT of the abdomen, were normal, and that he had a "viral syndrome." Several days later, and 5 weeks after the onset of symptoms, the development of dull right upper-quadrant pain and mild nausea without vomiting prompted the current presentation to the community hospital. He reported several years of loose stools, but denied rash, arthritis, diarrhea, neck stiffness, cough, or other complaints.

A detailed past medical, social, and family history is required, with particular attention to ethnicity; immunocompromising conditions such as splenectomy or corticosteroid use; undiagnosed febrile diseases; severe, unusual, or recurrent infections; medication use; diet; sexual history; pet exposures; and any personal or family history of cancer. The development of right upper-quadrant pain mandates attention to risk factors for viral hepatitis, known biliary pathology, or travel that might predispose the patient to pyogenic or amoebic liver abscess, and hematochezia, which could suggest a malignancy metastatic to the liver. Additionally, chronic diarrhea with new right upper-quadrant pain may represent inflammatory bowel disease complicated by primary sclerosing cholangitis (PSC).

The patient was a Caucasian male of Mediterranean ancestry with thalassemia minor. He had undergone dilation of a benign esophageal stricture, but no surgical procedures, and he had never experienced unexplained fever or unusual infections. Medication exposure was limited to occasional use of acetaminophen for fever, and he had no known allergies. His diet was unremarkable and included no well water or unpasteurized dairy products. He denied risk factors for tuberculosis. He drank 2 to 10 beers a day, 5 times a week, had last smoked 10 years previously, and had never used illicit drugs. He denied any high-risk sexual contacts and was monogamous with his wife, with whom he had 2 children. The family owned no pets and no relatives had suffered from malignant, rheumatologic, or febrile illness, with the exception of "hand, foot, and mouth infection" in an infant son, 1 year previously. The patient had never traveled outside of New England.

The history has uncovered several clues, but their relevance is doubtful. His ethnicity suggests possible familial Mediterranean fever, but recurrent abdominal pain and polyserositis, rather than a single prolonged episode, would be expected with this disease. A transfusion history should be obtained to explore the possibility of viral hepatitis. While iron overload can predispose patients to various infections including liver abscess, thalassemia minor should not require transfusion. Esophageal stricture could conceivably be due to histoplasmosis (complicated by mediastinal fibrosis) or tuberculosis, but is probably unrelated to his present illness. His excessive alcohol intake increases his risk for esophageal cancer and liver disease, but it is unlikely that metastatic disease to the liver would present with fever without preceding dysphagia, or that alcoholic hepatitis could have escaped detection after evaluations by several physicians.

We need to learn the details of the patient's physical examination. Given the development of right upper-quadrant pain, I would particularly like to know if he had hepatosplenomegaly and if a Murphy's sign was present.

His temperature ranged from $36.9^{\circ} \mathrm{C}$ to $39.8^{\circ} \mathrm{C}$, his pulse was 76 beats per minute with minimal elevations during fever spikes, and his respirations were 18 per minute. His blood pressure was 105/70 mm Hg. He was a well-developed, overweight male with scleral icterus. 
He had good dentition and an oropharynx free of lesions. Cardiac examination demonstrated a regular rhythm with a normal $\mathrm{S} 1$ and $\mathrm{S} 2$, without murmurs or peripheral stigmata of infectious endocarditis. A smooth, minimally tender liver edge was palpable $2 \mathrm{~cm}$ below the costal margin; the spleen was nonpalpable. Murphy's sign was absent. There was no lymphadenopathy or rash. He had multiple, shallow, uninfected lacerations of both hands in various stages of healing. The remainder of his examination was normal.

The patient has obvious liver involvement. The pulse-temperature dissociation suggests a variety of infections, including salmonellosis, psittacosis, typhoid fever, leptospirosis, tularemia, brucellosis, legionellosis, and mycoplasma pneumoniae infection. The patient should be asked how and when he injured his hands, as fresh water exposure can transmit leptospirosis across broken skin. However, while severe leptospirosis can cause fever and jaundice, the long duration of illness is not typical. The cryptogenic form of tularemia-which can manifest as a "typhoidal" illness—-should be considered, given that tularemia is present in the area the patient visited; he should be asked about exposure to rabbits.

At this point, I would like to see a standard biochemical profile, a liver panel, a complete blood count and differential, urinalysis, chest X-ray, and an electrocardiogram. I would examine thick and thin Wright-Giemsa-stained smears for evidence of babesiosis. Blood cultures should be held for at least 2 weeks to recover fastidious organisms like Francisella tularensis and Brucella sp. Bone marrow cultures should be obtained; they are more sensitive for mycobacteria and Brucella, and may also yield fungal pathogens. Serologies for a variety of infectious diseases, such as leptospirosis, typhoid fever, and tularemia, will be required if other diagnostic tests are unrevealing.

His white cell count was $8,100 / \mu \mathrm{L}$, with a normal differential, and his hemoglobin was $10 \mathrm{~g} /$ $\mathrm{dL}$ (normal range, 14-17), with a mean corpuscular hemoglobin of $63 \mu \mathrm{m}^{3}$ (normal range, 8298). The platelet count was $303,000 / \mu \mathrm{L}$. Serum electrolytes were normal. His aspartate aminotransferase was $58 \mathrm{U} / \mathrm{L}$ and his alanine aminotransferase was $60 \mathrm{U} / \mathrm{L}$ (normal range for both, 10-45). Bilirubin was $2.6 \mathrm{mg} / \mathrm{dL}$ (normal, <1.2); direct bilirubin was $0.9 \mathrm{mg} / \mathrm{dL}$. Alkaline phosphatase was $150 \mathrm{U} / \mathrm{L}$. Lactate dehydrogenase was $342 \mathrm{U} / \mathrm{L}$ (normal range, 22-51). A lipase was $62 \mathrm{U} / \mathrm{L}$. International normalized ratio (INR) was 1.4 with an activated partial thromboplastin time (aPTT) of 52 seconds (normal range, 25-33). Erythrocyte sedimentation rate (ESR) was $50 \mathrm{~mm} /$ hour (normal range, $0-15$ ). Iron studies showed a suppressed iron and iron-binding capacity and elevated haptoglobin and ferritin $(1878 \mathrm{ng} / \mu \mathrm{L}$; normal range, $22-$ 322). Several blood cultures obtained at admission showed no growth after 48 hours of incubation.

The anemia, low mean cell volume (MCV), and elevated ferritin and ESR are consistent with anemia of chronic disease, superimposed upon thalassemia minor. Transaminase elevations occur in a plethora of infectious processes. The elevated INR and aPTT are concerning, and may indicate a septic or malignant process with disseminated intravascular coagulation (DIC). While there is no mention of clinical DIC, it would be appropriate to obtain D-dimers, fibrin degradation products, and a fibrinogen level. The platelet count is normal, which is reassuring.

Before initiating any empiric antimicrobials, I would obtain an abdominal ultrasound, and possibly an abdominal CT. Hepatitis (especially B and C), cytomegalovirus, and Epstein-Barr virus serologies should be obtained. A variety of conditions including leptospirosis, tularemia, and babesiosis are possible; specific laboratory testing is required to guide therapy.

Ultrasound showed a thickened gallbladder; the liver was slightly enlarged with normal echotexture. Magnetic resonance cholangiopancreatography (MRCP) showed diffuse sequential beading and scarring of his extrahepatic biliary ducts. 
There is no evidence of biliary stones, intrahepatic tumor, or abscess to explain the fever and hepatitis, although it would be helpful to know what other abdominal structures were imaged. The MRCP finding increases my suspicion of PSC, possibly complicated by infection, although the biliary abnormalities may be incidental, and an unrelated process may be responsible for the clinical presentation.

His physicians considered the possibilities of PSC and cholangiopathy due to as-yet undiagnosed acquired immunodeficiency syndrome. Ampicillin-sulbactam, ceftazidime, and gentamicin were administered for possible bacterial cholangitis, and endoscopic retrograde cholangiopancreatography was performed. This procedure showed only slight narrowing of his common bile duct, which was felt to be a normal variant. He felt no better after several days of antibiotic therapy, and was transferred to a tertiary care center for further evaluation. Repeat physical exam and laboratory studies were essentially unchanged. The patient explained that his hand lacerations were sustained during his work as a butcher who worked with lamb, beef, rabbit, and poultry. He rarely wore protective gloves because they induced contact dermatitis.

Tularemia becomes more likely given his history of rabbit butchering. Salmonellosis and leptospirosis also remain possible. Typhoid fever and brucellosis are unlikely unless the patient worked with imported exotic animals. At this point, given the systemic illness, empiric antibacterial therapy is reasonable. Of the chosen antimicrobials, only the gentamicin would reliably treat tularemia. I would stop ampicillin-sulbactam and ceftazidime and replace gentamicin with ciprofloxacin, an effective and better-tolerated agent for tularemia. Cultures of blood and bone marrow aspirate should be obtained. Stool should be cultured for Salmonella. Tularemia, leptospirosis, and typhoid serologies should be sent to a reference laboratory. At this point in the patient's illness, high-titered antibodies should be present. However, it would be ideal to compare titers with those from previous serum sample, if possible.

The patient's antimicrobials were narrowed to doxycycline alone, for suspected zoonotic infection, but his fevers were unchanged after 1 week of treatment. Hepatitis serologies, human immunodeficiency virus (HIV) antibody, and smears for ehrlichiosis and babesiosis were negative. He had a positive immunoglobulin (Ig)G and a negative IgM for Epstein-Barr virus and cytomegalovirus. Tularemia, ehrlichiosis, leptospirosis, brucellosis, and Query fever (Q fever) serologies were ordered. The elevated aPTT did not correct when his serum was mixed with normal serum. Thrombin was normal; factor VIII, von Willebrand (VW) factor, and VW cofactor were mildly elevated. Lupus anticoagulant was detected. A hepatologist declined to obtain a liver biopsy, citing the elevated aPTT and pending serologies. Given his clinical stability, the patient was discharged on doxycycline to await further results.

My highest suspicion is for tularemia, and I would switch antibiotic treatment to ciprofloxacin, awaiting serological results. Some in vitro studies have suggested that $F$. tularensis may often be resistant to doxycycline, and recent clinical experience has shown fluoroquinolones are superior to doxycycline in the treatment of tularemia.

His serologic results were as follows: tularemia, 1:32 (positive, $\geq 1: 128$ ); ehrlichia, 1:128 (granulocytic) and <1:64 (monocytic; normal for both, <1:64); leptospira, agglutinated nonspecifically; Brucella IgG and IgM 1 (negative, <9), Q fever (coxiella) IgG $1+2$, IgM 1 +2 , all positive at 1:256 $(<1: 16)$. A transesophageal echocardiogram showed no evidence of endocarditis. The patient was treated with 10 weeks of doxycycline for $\mathrm{Q}$ fever hepatitis. His fever, headache, and laboratory abnormalities resolved, and he remained well after the completion of therapy.

The serologies suggest the patient had Coxiella burnetii hepatitis, and illustrate the value of a precise exposure history. Most butchers work only with muscle tissue and have a negligible 
risk of Q fever. In retrospect, it became clear that he worked part-time in a slaughterhouse, where highly infectious reproductive tract fluids can dry and aerosolize.

\section{Commentary}

Q fever was proposed as the name for a febrile illness affecting Australian slaughterhouse workers in $1937 .{ }^{1}$ The etiologic agent, C. burnetii, is a small, gram-negative, obligate intracellular proteobacterium that exists in 2 distinct phases, specializing either in entering or persisting in macrophage lysosomes. ${ }^{2}$ Additionally, spores are formed and can persist in soil.

Q fever is an uncommonly recognized disease, in part because most infected persons have no symptoms or mild symptoms.3 In the United States, the estimated annual incidence has been 0.28 per million (about 50 cases per year) since 1999 , when Q fever became a reportable disease due to bioterrorism concerns. In France, more frequent farming of goats and sheep may be responsible for the much higher annual incidence of 500 per million. ${ }^{4}$ Spread is usually occupational, via aerosol contact with the dried reproductive tract secretions of animals (mainly cattle, sheep, and goats), in a slaughterhouse or farm setting. However, wind-borne dust can carry spores long distances, and spread can occur from household pets, unpasteurized dairy products, laboratory work, and possibly ticks. 3 More than 30 cases have been reported in military personnel deployed to Iraq and Afghanistan, several without obvious exposures.5 One review noted a single reported case of intradermal inoculation, 3 making this patient's lacerations a possible site of infection, but he was also at risk for inhalational exposure-when he was later asked about the details of his work, he acknowledged working at a slaughterhouse as well as a supermarket.

Symptomatic patients are male in $77 \%$ of cases, can usually identify an occupational exposure, and have a mean age of 50 years. 4 Fever, which lasts 5 to 57 days, as well as fatigue and headaches, begin after a 1-week to 3-week incubation period. Atypical pneumonia or rash may occur; meningoencephalitis and myocarditis portend a worse prognosis. As with this patient, $45 \%$ to $85 \%$ of patients suffer from hepatitis, although few have an abnormal bilirubin.3 Liver biopsy usually reveals granulomas, which may have a classic "doughnut hole" appearance, 2 , 3 although this patient ultimately received a diagnosis without the procedure. Acute $\mathrm{Q}$ fever rarely $(\sim 5 \%)$ requires hospitalization, and fatalities are extremely rare. ${ }^{3}$

Chronic infection (ie, lasting >6 months) most often occurs as endocarditis, although chronic hepatitis, osteomyelitis, and infections of other sites occur. Interestingly, this patient's lupus anticoagulant may have been related to his underlying illness, as autoantibodies frequently occur in Q fever, especially in patients with hepatitis, many of whom develop smooth muscle antibodies, a positive Coombs test, antiprothrombinase, or other autoantibodies, ${ }^{3}$ and there is a high incidence of antiphospholipid antibodies, particularly anticardiolipin and lupus anticoagulant antibodies.6

Because C. burnetii is an obligate intracellular pathogen, culture requires either tissue or live animal inoculation, and the diagnosis is usually made serologically. Paired sera demonstrating seroconversion or a 4-fold increase in titers are most conclusive, but a single sample may be used. Anti-phase II antibodies are detectable in $90 \%$ of patients within 3 weeks of infection3 and peak at 2 months $^{5}$; this patient's phase II sera $(\operatorname{IgG}>1: 200, \operatorname{IgM}>1: 50)$ are said to be $100 \%$ predictive for acute $\mathrm{Q}$ fever. ${ }^{3}$ High-titer anti-phase I antibodies, in contrast, indicate chronic infection, and a titer $\geq 1: 800$ is one of the modified Duke criteria for endocarditis.5

Acute $\mathrm{Q}$ fever is generally treated with doxycycline for 14 days, although prolonged therapy may be advisable to prevent endocarditis if preexisting valvular lesions are present.2,5 Fluoroquinolones are another option and may be especially useful for meningoencephalitis.5 Because acute $\mathrm{Q}$ fever is generally self-limited, demonstrating a clear benefit to antibiotic 
therapy is difficult. The available evidence, which was largely obtained from $\mathrm{Q}$ fever pneumonia patients, suggests that tetracycline therapy shortens fever duration. ${ }^{3}$ Patients with $\mathrm{Q}$ fever hepatitis may have a protracted course. On the basis of anecdotal reports, some experts add prednisone (tapered from $40 \mathrm{mg}$ daily over a week) for patients with $\mathrm{Q}$ fever hepatitis who fail to respond to doxycycline promptly. ${ }^{3}$ While this patient's fever was unchanged after a week of therapy, he was well into his treatment course when his diagnosis was ultimately confirmed. His physicians felt that prednisone would be of uncertain benefit and opted not to administer it.

Treatment of Q fever endocarditis is often delayed by the combination of negative blood cultures and a low (12\%) rate of vegetation formation, increasing the risk of morbidity and mortality. ${ }^{3}$ Tetracycline monotherapy is associated with a greater than $50 \%$ risk of death,5 and even 4 years of treatment may fail to sterilize valve tissue. 3 However, if hydroxychloroquine is given with doxycycline for at least 18 months to alkalize lysosomes and improve bacterial killing, the mortality rate can be lowered to about 5\%.3,5 Patients should be warned of the risk of photosensitivity, and monitored for retinal toxicity ${ }^{2}$ and serologic evidence of relapse. 5

Before serologic results confirmed the diagnosis of Q fever, both the patient's clinicians and the discussant had to craft an antibiotic regimen for a suspected zoonosis. The patient received doxycycline, a good choice for leptospirosis, ${ }^{7}$ brucellosis,${ }^{8}$ tularemia, ${ }^{9}$ and Q fever, ${ }^{3}$ all possible after livestock exposure, as well as ehrlichiosis. ${ }^{10}$ The discussant, who suspected tularemia, worried about the possibility of doxycycline resistance and selected ciprofloxacin instead. While fluoroquinolones are probably superior to doxycycline for mild to moderate tularemia, ${ }^{11,12}$ amino-glycosides would be preferred for severe disease, ${ }^{9}$ and ciprofloxacin experience in leptospirosis ${ }^{7}$ and ehrlichiosis ${ }^{10}$ is limited. Neither selection would be optimal for brucellosis, for which either doxycycline or ciprofloxacin should be combined with another agent such as rifampin. ${ }^{8}$ The most reasonable empiric regimen is debatable, but in the absence of pathognomonic findings of tularemia, his treating physicians favored the broader activity of doxycycline.

Ultimately, the choice of antibiotics in this case hinged on the details of the patient's occupational exposures. His first 2 courses of antibiotics were based not on his exposure history, but on radiographic findings that were later proven spurious. The regimens selected by the discussant and by physicians at the referral hospital both targeted pathogens suggested by the patient's occupational history instead, but both were missing parts of the puzzle as well. The discussant thought the patient performed commercial butcher-shop work, which is only rarely ${ }^{13}$ mentioned in the context of $Q$ fever transmission. Several of the admitting physicians at the referral hospital were unaware of the importance of the butcher/slaughterhouse-worker distinction. Physicians need a detailed understanding of both the exposure history and the biology of possible pathogens to craft an optimal differential diagnosis and empiric antibiotic regimen.

On the other hand, in most patients with fever of unknown origin (FUO; ie, >3 weeks with temperature $>38.3^{\circ}$ on multiple occasions, without a diagnosis after a weeklong evaluation), 14 empiric antibiotic therapy is rarely a wise intervention. Clinicians should avoid blind administration of antibiotics as a diagnostic tool, given the inability to distinguish clinical responses from spontaneous resolution, or pinpoint a specific cause and thus a precise treatment plan and duration. However, empiric tetracyclines have been employed when intracellular pathogens were a suspected cause of FUO, as in one series of French patients in which Q fever was common.15 In this patient's case, no specific finding pointed to $\mathrm{Q}$ fever before the serologies became available, but the rare infections considered in this case can be considered "doxycycline-deficient states," meaning that empiric tetracycline therapy often leads to improvement. Recognizing "doxycycline deficiency" can guide therapy while definitive results 
are pending, and empiric doxycycline is particularly important if potentially aggressive zoonoses, such as Rocky Mountain spotted fever, are suspected.

\section{Teaching Points}

- A detailed and precise exposure history is crucial for the diagnosis of Q fever and other zoonoses and for the individualized evaluation of FUO in general.

- $\mathrm{Q}$ fever is a rare disease that most commonly causes undifferentiated fever, pneumonia, hepatitis, and when chronic, often reflects endovascular infection, which is frequently difficult to eradicate.

- Doxycycline is effective for many, but not all zoonoses (babesia is a notable exception). Empiric therapy is reasonable if suspicion is high.

\section{References}

1. Derrick EH. "Q" fever, new fever entity: clinical features, diagnosis and laboratory investigation. Med J Aust 1937;2:281-299.

2. Parker NR, Barralet JH, Bell AM. Q fever. Lancet 2006;367(9511):679-688. [PubMed: 16503466]

3. Maurin M, Raoult D. Q fever. Clin Microbiol Rev 1999;12:518-553. [PubMed: 10515901]

4. McQuiston JH, Holman RC, McCall CL, et al. National surveillance and the epidemiology of human Q fever in the United States, 1978-2004. Am J Trop Med Hyg 2006;75:36-40. [PubMed: 16837706]

5. Hartzell JD, Wood-Morris RN, Martinez LJ, Trotta RF. Q fever: epidemiology, diagnosis, and treatment. Mayo Clin Proc 2008;83(5):574-579. [PubMed: 18452690]

6. Ordi-Ros J, Selva-O'Callaghan A, Monegal-Ferran F, et al. Prevalence, significance, and specificity of antibodies to phospholipids in Q fever. Clin Infect Dis 1994;18:213-218. [PubMed: 8161629]

7. Griffith ME, Hospenthal DR, Murray CK. Antimicrobial therapy of leptospirosis. Curr Opin Infect Dis 2006;19:533-537. [PubMed: 17075327]

8. Ariza J, Gudiol F, Pallares R, et al. Treatment of human brucellosis with doxycycline plus rifampin or doxycycline plus streptomycin. A randomized, double-blind study. Ann Intern Med 1992;117:25-30. [PubMed: 1596044]

9. Eliasson H, Broman T, Forsman M, Bäck E. Tularemia: current epidemiology and disease management. Infect Dis Clin North Am 2006;20:289-311. ix. [PubMed: 16762740]

10. Dumler JS, Madigan JE, Pusterla N, Bakken JS. Ehrlichioses in humans: epidemiology, clinical presentation, diagnosis, and treatment. Clin Infect Dis 2007;45(Suppl 1):S45-S51. [PubMed: 17582569]

11. Tärnvik A, Chu MC. New approaches to diagnosis and therapy of tularemia. Ann NY Acad Sci 2007;1105:378-404. [PubMed: 17468229]

12. Meric M, Wilke A, Finke EJ, et al. Evaluation of clinical, laboratory, and therapeutic features of 145 tularemia cases: the role of quinolones in oropharyngeal tularemia. APMIS 2008;116:66-73. [PubMed: 18254782]

13. Kourany M, Johnson KM. A survey of Q fever antibodies in a high risk population in Panamá. Am J Trop Med Hyg 1980;29(5):1007-1011. [PubMed: 7435778]

14. Arnow PM, Flaherty JP. Fever of unknown origin. Lancet 1997;350:575-580. [PubMed: 9284789]

15. Zenone T. Fever of unknown origin in adults: evaluation of 144 cases in a non-university hospital. Scand J Infect Dis 2006;38:632-638. [PubMed: 16857607] 